\title{
Summing Formulas for Generalized Tribonacci Numbers
}

\author{
Yüksel Soykan $^{1}$ \\ ${ }^{1}$ Department of Mathematics, Faculty of Science and Arts, Zonguldak Bülent Ecevit University, Zonguldak, Turkey
}

\section{Article Info}

Keywords: Tribonacci numbers, Padovan numbers, Perrin numbers, Sum formulas, Summing formulas 2010 AMS: 11B37, 11B39, $11 B 83$

Received: 24 October 2019

Accepted: 11 December 2019 Available online: 25 March 2020

\begin{abstract}
In this paper, closed forms of the summation formulas for generalized Tribonacci numbers are presented. Then, some previous results are recovered as particular cases of the present results. As special cases, we give summation formulas of Tribonacci, Tribonacci-Lucas, Padovan, Perrin, Narayana and some other third order linear recurrance sequences. All the summing formulas of well known recurrence sequences which we deal with are linear except the cases Pell-Padovan and Padovan-Perrin.
\end{abstract}

\section{Introduction}

In this work, we investigate linear summation formulas of generalized Tribonacci numbers. Some summing formulas of the Pell and Pell-Lucas numbers are well known and given in [11, 12], see also [9]. For linear sums of Fibonacci, Tribonacci, Tetranacci, Pentanacci and Hexanacci numbers, see [10,24], [8,16], [21, 31], [22], and [23] respectively. First, in this section, we present some background about generalized Tribonacci numbers. The generalized Tribonacci sequence $\left\{W_{n}\left(W_{0}, W_{1}, W_{2} ; r, s, t\right)\right\}_{n \geq 0}$ (or shortly $\left.\left\{W_{n}\right\}_{n \geq 0}\right)$ is defined as follows:

$$
W_{n}=r W_{n-1}+s W_{n-2}+t W_{n-3}, \quad W_{0}=a, W_{1}=b, W_{2}=c, n \geq 3
$$

where $W_{0}, W_{1}, W_{2}$ are arbitrary complex numbers and $r, s, t$ are real numbers. The generalized Tribonacci sequence has been studied by many authors, see for example $[2,3,5,7,14,15,17,18,19,26,27,28,29,30]$.

The sequence $\left\{W_{n}\right\}_{n \geq 0}$ can be extended to negative subscripts by defining

$$
W_{-n}=-\frac{s}{t} W_{-(n-1)}-\frac{r}{t} W_{-(n-2)}+\frac{1}{t} W_{-(n-3)}
$$

for $n=1,2,3, \ldots$ when $t \neq 0$. Therefore, recurrence (1.1) holds for all integer $n$.

If we set $r=s=t=1$ and $W_{0}=0, W_{1}=1, W_{2}=1$ then $\left\{W_{n}\right\}$ is the well-known Tribonacci sequence and if we set $r=s=t=1$ and $W_{0}=3, W_{1}=1, W_{2}=3$ then $\left\{W_{n}\right\}$ is the well-known Tribonacci-Lucas sequence.

In fact, the generalized Tribonacci sequence is the generalization of the well-known sequences like Tribonacci, Tribonacci-Lucas, Padovan (Cordonnier), Perrin, Padovan-Perrin, Narayana, third order Jacobsthal and third order Jacobsthal-Lucas. In literature, for example, the following names and notations (see Table 1) are used for the special case of $r, s, t$ and initial values. 


\begin{tabular}{ccc}
\hline Sequences (Numbers) & Notation & OEIS [20] \\
\hline Tribonacci & $\left\{T_{n}\right\}=\left\{W_{n}(0,1,1 ; 1,1,1)\right\}$ & A000073, A057597 \\
Tribonacci-Lucas & $\left\{K_{n}\right\}=\left\{W_{n}(3,1,3 ; 1,1,1)\right\}$ & A001644, A073145 \\
third order Pell & $\left\{P_{n}^{(3)}\right\}=\left\{W_{n}(0,1,2 ; 2,1,1)\right\}$ & A077939, A077978 \\
third order Pell-Lucas & $\left\{Q_{n}^{(3)}\right\}=\left\{W_{n}(3,2,6 ; 2,1,1)\right\}$ & A276225, A276228 \\
third order modified Pell & $\left\{E_{n}^{(3)}\right\}=\left\{W_{n}(0,1,1 ; 2,1,1)\right\}$ & A077997, A078049 \\
Padovan (Cordonnier) & $\left\{P_{n}\right\}=\left\{W_{n}(1,1,1 ; 0,1,1)\right\}$ & A000931 \\
Perrin (Padovan-Lucas) & $\left\{E_{n}\right\}=\left\{W_{n}(3,0,2 ; 0,1,1)\right\}$ & A001608, A078712 \\
Padovan-Perrin & $\left\{S_{n}\right\}=\left\{W_{n}(0,0,1 ; 0,1,1)\right\}$ & A000931, A176971 \\
Pell-Padovan & $\left\{R_{n}\right\}=\left\{W_{n}(1,1,1 ; 0,2,1)\right\}$ & A066983, A128587 \\
Pell-Perrin & $\left\{C_{n}\right\}=\left\{W_{n}(3,0,2 ; 0,2,1)\right\}$ & \\
Jacobsthal-Padovan & $\left\{Q_{n}\right\}=\left\{W_{n}(1,1,1 ; 0,1,2)\right\}$ & A159284 \\
Jacobsthal-Perrin (-Lucas) & $\left\{D_{n}\right\}=\left\{W_{n}(3,0,2 ; 0,1,2)\right\}$ & A072328 \\
Narayana & $\left\{N_{n}\right\}=\left\{W_{n}(0,1,1 ; 1,0,1)\right\}$ & A078012 \\
third order Jacobsthal & $\left\{J_{n}^{(3)}\right\}=\left\{W_{n}(0,1,1 ; 1,1,2)\right\}$ & A077947 \\
third order Jacobsthal-Lucas & $\left\{j_{n}^{(3)}\right\}=\left\{W_{n}(2,1,5 ; 1,1,2)\right\}$ & A226308 \\
\hline
\end{tabular}

Table 1: A few special case of generalized Tribonacci sequences

Note that the sequence $\left\{C_{n}\right\}$ is't in the database of http://oeis.org [20], yet.

\section{Sum formulas of Generalized Tribonacci Numbers with Positive Subscripts}

The following Theorem presents some linear summing formulas of generalized Tribonacci numbers with positive subscripts.

Theorem 2.1. For $n \geq 0$, we have the following formulas:

(a) (Sum of the generalized Tribonacci numbers) If $r+s+t-1 \neq 0$, then

$$
\sum_{k=0}^{n} W_{k}=\frac{W_{n+3}+(1-r) W_{n+2}+(1-r-s) W_{n+1}-W_{2}+(r-1) W_{1}+(r+s-1) W_{0}}{r+s+t-1} .
$$

(b) If $2 s+2 r t+r^{2}-s^{2}+t^{2}-1=(r+s+t-1)(r-s+t+1) \neq 0$ then

$$
\sum_{k=0}^{n} W_{2 k}=\frac{(-s+1) W_{2 n+2}+(t+r s) W_{2 n+1}+\left(t^{2}+r t\right) W_{2 n}+(-1+s) W_{2}+(-t-r s) W_{1}+\left(-1+r^{2}-s^{2}+r t+2 s\right) W_{0}}{(r+s+t-1)(r-s+t+1)}
$$

and

$$
\sum_{k=0}^{n} W_{2 k+1}=\frac{(r+t) W_{2 n+2}+\left(s-s^{2}+t^{2}+r t\right) W_{2 n+1}+(t-s t) W_{2 n}+(-r-t) W_{2}+\left(-1+s+r^{2}+r t\right) W_{1}+(-t+s t) W_{0}}{(r-s+t+1)(r+s+t-1)} .
$$

(c) If $r+t \neq 0, s=1$ then

$$
\sum_{k=0}^{n} W_{2 k}=\frac{1}{r+t}\left(W_{2 n+1}+t W_{2 n}-W_{1}+r W_{0}\right)
$$

and

$$
\sum_{k=0}^{n} W_{2 k+1}=\frac{1}{r+t}\left(W_{2 n+2}+t W_{2 n+1}-W_{2}+r W_{1}\right) .
$$

Note that (c) is a special case of (b).

Proof.

(a) Using the recurrence relation

$$
W_{n}=r W_{n-1}+s W_{n-2}+t W_{n-3}
$$

i.e.

$$
t W_{n-3}=W_{n}-r W_{n-1}-s W_{n-2}
$$

we obtain

$$
\begin{aligned}
t W_{0} & =W_{3}-r W_{2}-s W_{1} \\
t W_{1} & =W_{4}-r W_{3}-s W_{2} \\
t W_{2} & =W_{5}-r W_{4}-s W_{3} \\
& \vdots \\
t W_{n-1} & =W_{n+2}-r W_{n+1}-s W_{n} \\
t W_{n} & =W_{n+3}-r W_{n+2}-s W_{n+1} .
\end{aligned}
$$


If we add the equations by side by, we get

$$
\sum_{k=0}^{n} W_{k}=\frac{W_{n+3}+(1-r) W_{n+2}+(1-r-s) W_{n+1}-W_{2}+(r-1) W_{1}+(r+s-1) W_{0}}{r+s+t-1} .
$$

(b) and (c) Using the recurrence relation

$$
W_{n}=r W_{n-1}+s W_{n-2}+t W_{n-3}
$$

i.e.

$$
r W_{n-1}=W_{n}-s W_{n-2}-t W_{n-3}
$$

we obtain

$$
\begin{aligned}
r W_{3} & =W_{4}-s W_{2}-t W_{1} \\
r W_{5} & =W_{6}-s W_{4}-t W_{3} \\
\vdots & \\
r W_{2 n+1} & =W_{2 n+2}-s W_{2 n}-t W_{2 n-1} . \\
r W_{2 n+3} & =W_{2 n+4}-s W_{2 n+2}-t W_{2 n+1}
\end{aligned}
$$

Now, if we add the above equations by side by, we get

$$
r\left(-W_{1}+\sum_{k=0}^{n} W_{2 k+1}\right)=\left(W_{2 n+2}-W_{2}-W_{0}+\sum_{k=0}^{n} W_{2 k}\right)-s\left(-W_{0}+\sum_{k=0}^{n} W_{2 k}\right)-t\left(-W_{2 n+1}+\sum_{k=0}^{n} W_{2 k+1}\right) .
$$

Similarly, using the recurrence relation

$$
W_{n}=r W_{n-1}+s W_{n-2}+t W_{n-3}
$$

i.e.

$$
r W_{n-1}=W_{n}-s W_{n-2}-t W_{n-3}
$$

we write the following obvious equations;

$$
\begin{aligned}
r W_{2} & =W_{3}-s W_{1}-t W_{0} \\
r W_{4} & =W_{5}-s W_{3}-t W_{2} \\
r W_{6} & =W_{7}-s W_{5}-t W_{4} \\
\vdots & \\
r W_{2 n} & =W_{2 n+1}-s W_{2 n-1}-t W_{2 n-2} \\
r W_{2 n+2} & =W_{2 n+3}-s W_{2 n+1}-t W_{2 n} .
\end{aligned}
$$

Now, if we add the above equations by side by, we obtain

$$
r\left(-W_{0}+\sum_{k=0}^{n} W_{2 k}\right)=\left(-W_{1}+\sum_{k=0}^{n} W_{2 k+1}\right)-s\left(-W_{2 n+1}+\sum_{k=0}^{n} W_{2 k+1}\right)-t\left(-W_{2 n}+\sum_{k=0}^{n} W_{2 k}\right) .
$$

Then, solving the system (2.1)-(2.2), the required results of (b) and (c) follow.

For another proof (using mathematical induction) of the formula in Theorem 2.1 (a), see [4].

Taking $r=s=t=1$ in Theorem 2.1 (a) and (b) (or (c)), we obtain the following Proposition.

Proposition 2.2. If $r=s=t=1$ then for $n \geq 0$ we have the following formulas:

(a) $\sum_{k=0}^{n} W_{k}=\frac{1}{2}\left(W_{n+3}-W_{n+1}-W_{2}+W_{0}\right)$.

(b) $\sum_{k=0}^{n} W_{2 k}=\frac{1}{2}\left(W_{2 n+1}+W_{2 n}-W_{1}+W_{0}\right)$.

(c) $\sum_{k=0}^{n} W_{2 k+1}=\frac{1}{2}\left(W_{2 n+2}+W_{2 n+1}-W_{2}+W_{1}\right)$.

From the above Proposition, we have the following Corollary which gives linear sum formulas of Tribonacci numbers (take $W_{n}=T_{n}$ with $\left.T_{0}=0, T_{1}=1, T_{2}=1\right)$.

Corollary 2.3. [8,16]For $n \geq 0$, Tribonacci numbers have the following properties.

(a) $\sum_{k=0}^{n} T_{k}=\frac{1}{2}\left(T_{n+3}-T_{n+1}-1\right)$.

(b) $\sum_{k=0}^{n} T_{2 k}=\frac{1}{2}\left(T_{2 n+1}+T_{2 n}-1\right)$.

(c) $\sum_{k=0}^{n} T_{2 k+1}=\frac{1}{2}\left(T_{2 n+2}+T_{2 n+1}\right)$.

Taking $W_{n}=K_{n}$ with $K_{0}=3, K_{1}=1, K_{2}=3$ in the above Proposition, we have the following Corollary which presents linear sum formulas of Tribonacci-Lucas numbers. 
Corollary 2.4. $[8,16]$ For $n \geq 0$, Tribonacci-Lucas numbers have the following properties.

(a) $\sum_{k=0}^{n} K_{k}=\frac{1}{2}\left(K_{n+3}-K_{n+1}\right)$.

(b) $\sum_{k=0}^{n} K_{2 k}=\frac{1}{2}\left(K_{2 n+1}+K_{2 n}+2\right)$.

(c) $\sum_{k=0}^{n} K_{2 k+1}=\frac{1}{2}\left(K_{2 n+2}+K_{2 n+1}-2\right)$.

Taking $r=2, s=1, t=1$ in Theorem 2.1 (a) and (b) (or (c)), we obtain the following Proposition.

Proposition 2.5. [25]If $r=2, s=1, t=1$ then for $n \geq 0$ we have the following formulas:

(a) $\sum_{k=0}^{n} W_{k}=\frac{1}{3}\left(W_{n+3}-W_{n+2}-2 W_{n+1}-W_{2}+W_{1}+2 W_{0}\right)$.

(b) $\sum_{k=0}^{n} W_{2 k}=\frac{1}{3}\left(W_{2 n+1}+W_{2 n}-W_{1}+2 W_{0}\right)$.

(c) $\sum_{k=0}^{n} W_{2 k+1}=\frac{1}{3}\left(W_{2 n+2}+W_{2 n+1}-W_{2}+2 W_{1}\right)$.

From the last Proposition, we have the following Corollary which gives linear sum formulas of third-order Pell numbers (take $W_{n}=P_{n}^{(3)}$ with $\left.P_{0}^{(3)}=0, P_{1}^{(3)}=1, P_{2}^{(3)}=2\right)$.

Corollary 2.6. [25]For $n \geq 0$, third-order Pell numbers have the following properties:

(a) $\sum_{k=0}^{n} P_{k}^{(3)}=\frac{1}{3}\left(P_{n+3}^{(3)}-P_{n+2}^{(3)}-2 P_{n+1}^{(3)}-1\right)$.

(b) $\sum_{k=0}^{n} P_{2 k}^{(3)}=\frac{1}{3}\left(P_{2 n+1}^{(3)}+P_{2 n}^{(3)}-1\right)$.

(c) $\sum_{k=0}^{n} P_{2 k+1}^{(3)}=\frac{1}{3}\left(P_{2 n+2}^{(3)}+P_{2 n+1}^{(3)}\right)$.

Taking $W_{n}=Q_{n}^{(3)}$ with $Q_{0}^{(3)}=3, Q_{1}^{(3)}=2, Q_{2}^{(3)}=6$ in the last Proposition, we have the following Corollary which presents linear sum formulas of third-order Pell-Lucas numbers.

Corollary 2.7. [25]For $n \geq 0$, third-order Pell-Lucas numbers have the following properties:

(a) $\sum_{k=0}^{n} Q_{k}^{(3)}=\frac{1}{3}\left(Q_{n+3}^{(3)}-Q_{n+2}^{(3)}-2 Q_{n+1}^{(3)}+2\right)$.

(b) $\sum_{k=0}^{n} Q_{2 k}^{(3)}=\frac{1}{3}\left(Q_{2 n+1}^{(3)}+Q_{2 n}^{(3)}+4\right)$.

(c) $\sum_{k=0}^{n} Q_{2 k+1}^{(3)}=\frac{1}{3}\left(Q_{2 n+2}^{(3)}+Q_{2 n+1}^{(3)}-2\right)$.

From the last Proposition, we have the following Corollary which gives linear sum formulas of third-order modified Pell numbers (take $W_{n}=E_{n}^{(3)}$ with $\left.E_{0}^{(3)}=0, E_{1}^{(3)}=1, E_{2}^{(3)}=1\right)$.

Corollary 2.8. [25]For $n \geq 0$, third-order modified Pell numbers have the following properties:

(a) $\sum_{k=0}^{n} E_{k}^{(3)}=\frac{1}{3}\left(E_{n+3}^{(3)}-E_{n+2}^{(3)}-2 E_{n+1}^{(3)}\right)$.

(b) $\sum_{k=0}^{n} E_{2 k}^{(3)}=\frac{1}{3}\left(E_{2 n+1}^{(3)}+E_{2 n}^{(3)}-1\right)$.

(c) $\sum_{k=0}^{n} E_{2 k+1}^{(3)}=\frac{1}{3}\left(E_{2 n+2}^{(3)}+E_{2 n+1}^{(3)}+1\right)$.

Taking $r=0, s=1, t=1$ in Theorem 2.1 (a) and (b) (or (c)), we obtain the following Proposition.

Proposition 2.9. If $r=0, s=1, t=1$ then for $n \geq 0$ we have the following formulas:

(a) $\sum_{k=0}^{n} W_{k}=W_{n+3}+W_{n+2}-W_{2}-W_{1}$.

(b) $\sum_{k=0}^{n} W_{2 k}=W_{2 n+1}+W_{2 n}-W_{1}$.

(c) $\sum_{k=0}^{n} W_{2 k+1}=W_{2 n+2}+W_{2 n+1}-W_{2}$.

From the last Proposition, we have the following Corollary which gives linear sum formulas of Padovan numbers (take $W_{n}=P_{n}$ with $\left.P_{0}=1, P=1, P_{2}=1\right)$.

Corollary 2.10. [1] For $n \geq 0$, Padovan numbers have the following properties.

(a) $\sum_{k=0}^{n} P_{k}=P_{n+3}+P_{n+2}-2$.

(b) $\sum_{k=0}^{n} P_{2 k}=P_{2 n+1}+P_{2 n}-1$.

(c) $\sum_{k=0}^{n} P_{2 k+1}=P_{2 n+2}+P_{2 n+1}-1$.

Taking $W_{n}=E_{n}$ with $E_{0}=3, E_{2}=0, E_{2}=2$ in the last Proposition, we have the following Corollary which presents linear sum formulas of Perrin numbers.

Corollary 2.11. [1] For $n \geq 0$, Perrin numbers have the following properties.

(a) $\sum_{k=0}^{n} E_{k}=E_{n+3}+E_{n+2}-2$.

(b) $\sum_{k=0}^{n} E_{2 k}=E_{2 n+1}+E_{2 n}$.

(c) $\sum_{k=0}^{n} E_{2 k+1}=E_{2 n+2}+E_{2 n+1}-2$.

Taking $W_{n}=S_{n}$ with $S_{0}=0, S_{2}=0, S_{2}=1$ in the last Proposition, we have the following Corollary which gives linear sum formulas of Padovan-Perrin numbers.

Corollary 2.12. For $n \geq 0$, Padovan-Perrin numbers have the following properties. 
(a) $\sum_{k=0}^{n} S_{k}=S_{n+3}+S_{n+2}-1$.

(b) $\sum_{k=0}^{n} S_{2 k}=S_{2 n+1}+S_{2 n}$.

(c) $\sum_{k=0}^{n} S_{2 k+1}=S_{2 n+2}+S_{2 n+1}-1$.

If $r=0, s=2, t=1$ then $(r-s+t+1)=0$ so we can't use Theorem 2.1 (b). In other words, the method of the proof Theorem 2.1 (b) can't be used to find $\sum_{k=0}^{n} W_{2 k}$ and $\sum_{k=0}^{n} W_{2 k+1}$. Therefore we need another method to find them which is given in the following Theorem.

Theorem 2.13. If $r=0, s=2, t=1$ then for $n \geq 0$ we have the following formulas:

(a) $\sum_{k=0}^{n} W_{k}=\frac{1}{2}\left(W_{n+3}+W_{n+2}-W_{n+1}-W_{2}-W_{1}+W_{0}\right)$.

(b) $\sum_{k=0}^{n} W_{2 k}=W_{2 n+1}+\left(W_{2}-W_{1}-W_{0}\right) n+W_{0}-W_{1}$.

(c) $\sum_{k=0}^{n} W_{2 k+1}=\frac{1}{2}\left(W_{2 n+3}+W_{2 n+2}-W_{2 n+1}+2 n\left(-W_{2}+W_{1}+W_{0}\right)-W_{2}+W_{1}-W_{0}\right)$.

Proof.

(a) Taking $r=0, s=2, t=1$ in Theorem 2.1 (a) we obtain (a).

(b) and (c) Using the recurrence relation

$$
W_{n}=2 W_{n-2}+W_{n-3}
$$

we obtain

$$
\begin{aligned}
\sum_{k=0}^{0} W_{2 k} & =W_{0} \\
\sum_{k=0}^{1} W_{2 k} & =W_{0}+W_{2}=W_{3}+W_{2}-2 W_{1} \\
\sum_{k=0}^{2} W_{2 k} & =W_{0}+W_{2}+W_{4}=W_{5}+2 W_{2}-3 W_{1}-W_{0} \\
\vdots & \\
\sum_{k=0}^{n} W_{2 k} & =W_{2 n+1}+\left(W_{2}-W_{1}-W_{0}\right) n+W_{0}-W_{1} .
\end{aligned}
$$

This result can be also proved by mathematical induction. Note that from (a) we get

$$
\sum_{k=0}^{n} W_{2 k+1}=\frac{1}{2}\left(W_{2 n+3}+W_{2 n+2}+W_{2 n+1}-W_{2}-W_{1}+W_{0}\right)-\sum_{k=0}^{n} W_{2 k} .
$$

Now, (c) follows from the last equation.

From the above Theorem we have the following Corollary which gives sum formulas of Pell-Padovan numbers (take $W_{n}=R_{n}$ with $\left.R_{0}=1, R_{1}=1, R_{2}=1\right)$.

Corollary 2.14. For $n \geq 0$, Pell-Padovan numbers have the following property:

(a) $\sum_{k=0}^{n} R_{k}=\frac{1}{2}\left(R_{n+3}+R_{n+2}-R_{n+1}-1\right)$.

(b) $\sum_{k=0}^{n} R_{2 k}=R_{2 n+1}-n$.

(c) $\sum_{k=0}^{n} R_{2 k+1}=\frac{1}{2}\left(R_{2 n+3}+R_{2 n+2}-R_{2 n+1}+2 n-1\right)$.

Taking $W_{n}=C_{n}$ with $C_{0}=3, C_{1}=0, C_{2}=2$ in the last Theorem, we have the following Corollary which presents sum formulas of Pell-Perrin numbers.

Corollary 2.15. For $n \geq 0$, Pell-Perrin numbers have the following property:

(a) $\sum_{k=0}^{n} C_{k}=\frac{1}{2}\left(C_{n+3}+C_{n+2}-C_{n+1}+1\right)$.

(b) $\sum_{k=0}^{n} C_{2 k}=C_{2 n+1}-n+3$.

(c) $\sum_{k=0}^{n} C_{2 k+1}=\frac{1}{2}\left(C_{2 n+3}+C_{2 n+2}-C_{2 n+1}+2 n-5\right)$.

Taking $r=0, s=1, t=2$ in Theorem 2.1 (a) and (b) (or (c)), we obtain the following Proposition.

Proposition 2.16. If $r=0, s=1, t=2$ then for $n \geq 0$ we have the following formulas:

(a) $\sum_{k=0}^{n} W_{k}=\frac{1}{2}\left(W_{n+3}+W_{n+2}-W_{2}-W_{1}\right)$.

(b) $\sum_{k=0}^{n} W_{2 k}=\frac{1}{2}\left(W_{2 n+1}+2 W_{2 n}-W_{1}\right)$.

(c) $\sum_{k=0}^{n} W_{2 k+1}=\frac{1}{2}\left(W_{2 n+2}+2 W_{2 n+1}-W_{2}\right)$.

Taking $W_{n}=Q_{n}$ with $Q_{0}=1, Q_{1}=1, Q_{2}=1$ in the last Proposition, we have the following Corollary which presents linear sum formulas of Jacobsthal-Padovan numbers.

Corollary 2.17. For $n \geq 0$, Jacobsthal-Padovan numbers have the following properties.

(a) $\sum_{k=0}^{n} Q_{k}=\frac{1}{2}\left(Q_{n+3}+Q_{n+2}-2\right)$. 
(b) $\sum_{k=0}^{n} Q_{2 k}=\frac{1}{2}\left(Q_{2 n+1}+2 Q_{2 n}-1\right)$.

(c) $\sum_{k=0}^{n} Q_{2 k+1}=\frac{1}{2}\left(Q_{2 n+2}+2 Q_{2 n+1}-1\right)$.

From the last Proposition, we have the following Corollary which gives linear sum formulas of Jacobsthal-Perrin numbers (take $W_{n}=D_{n}$ with $D_{0}=3, D_{1}=0, D_{2}=2$ ).

Corollary 2.18. For $n \geq 0$, Jacobsthal-Perrin numbers have the following properties.

(a) $\sum_{k=0}^{n} D_{k}=\frac{1}{2}\left(D_{n+3}+D_{n+2}-2\right)$.

(b) $\sum_{k=0}^{n} D_{2 k}=\frac{1}{2}\left(D_{2 n+1}+2 D_{2 n}\right)$.

(c) $\sum_{k=0}^{n} D_{2 k+1}=\frac{1}{2}\left(D_{2 n+2}+2 D_{2 n+1}-2\right)$.

Taking $r=1, s=0, t=1$ in Theorem 2.1 (a) and (c), we obtain the following Proposition.

Proposition 2.19. If $r=1, s=0, t=1$ then for $n \geq 0$ we have the following formulas:

(a) $\sum_{k=0}^{n} W_{k}=W_{n+3}-W_{2}$.

(b) $\sum_{k=0}^{n} W_{2 k}=\frac{1}{3}\left(W_{2 n+2}+W_{2 n+1}+2 W_{2 n}-W_{2}-W_{1}+W_{0}\right)$.

(c) $\sum_{k=0}^{n} W_{2 k+1}=\frac{1}{3}\left(2 W_{2 n+2}+2 W_{2 n+1}+W_{2 n}-2 W_{2}+W_{1}-W_{0}\right)$.

From the last Proposition, we have the following Corollary which presents linear sum formulas of Narayana numbers (take $W_{n}=N_{n}$ with $\left.N_{0}=0, N_{1}=1, N_{2}=1\right)$.

Corollary 2.20. For $n \geq 0$, Narayana numbers have the following properties.

(a) $\sum_{k=0}^{n} N_{k}=N_{n+3}-1$.

(b) $\sum_{k=0}^{n} N_{2 k}=\frac{1}{3}\left(N_{2 n+2}+N_{2 n+1}+2 N_{2 n}-2\right)$.

(c) $\sum_{k=0}^{n} N_{2 k+1}=\frac{1}{3}\left(2 N_{2 n+2}+2 N_{2 n+1}+N_{2 n}-1\right)$.

Taking $r=1, s=1, t=2$ in Theorem 2.1 (a) and (c), we obtain the following Proposition.

Proposition 2.21. If $r=1, s=1, t=2$ then for $n \geq 0$ we have the following formulas:

(a) $\sum_{k=0}^{n} W_{k}=\frac{1}{3}\left(W_{n+3}-W_{n+1}-W_{2}+W_{0}\right)$.

(b) $\sum_{k=0}^{n} W_{2 k}=\frac{1}{3}\left(W_{2 n+1}+2 W_{2 n}-W_{1}+W_{0}\right)$.

(c) $\sum_{k=0}^{n} W_{2 k+1}=\frac{1}{3}\left(W_{2 n+2}+2 W_{2 n+1}-W_{2}+W_{1}\right)$.

Taking $W_{n}=J_{n}^{(3)}$ with $J_{0}^{(3)}=0, J_{1}^{(3)}=1, J_{2}^{(3)}=1$ in the last Proposition, we have the following Corollary which presents linear sum formulas of third order Jacobsthal numbers.

Corollary 2.22. For $n \geq 0$, third order Jacobsthal numbers have the following properties.

(a) $[6] \sum_{k=0}^{n} J_{k}^{(3)}=\frac{1}{3}\left(J_{n+3}^{(3)}-J_{n+1}^{(3)}-1\right)$.

(b) $\sum_{k=0}^{n} J_{2 k}^{(3)}=\frac{1}{3}\left(J_{2 n+1}^{(3)}+2 J_{2 n}^{(3)}-1\right)$.

(c) $\sum_{k=0}^{n} J_{2 k+1}^{(3)}=\frac{1}{3}\left(J_{2 n+2}^{(3)}+2 J_{2 n+1}^{(3)}\right)$.

From the last Proposition, we have the following Corollary which gives linear sum formulas of third order Jacobsthal-Lucas numbers (take $W_{n}=j_{n}$ with $\left.j_{0}^{(3)}=2, j_{1}^{(3)}=1, j_{2}^{(3)}=5\right)$.

Corollary 2.23. For $n \geq 0$, third order Jacobsthal-Lucas numbers have the following properties.

(a) $[6] \sum_{k=0}^{n} j_{k}^{(3)}=\frac{1}{3}\left(j_{n+3}^{(3)}-j_{n+1}^{(3)}-3\right)$.

(b) $\sum_{k=0}^{n} j_{2 k}^{(3)}=\frac{1}{3}\left(j_{2 n+1}^{(3)}+2 j_{2 n}^{(3)}+1\right)$.

(c) $\sum_{k=0}^{n} j_{2 k+1}^{(3)}=\frac{1}{3}\left(j_{2 n+2}^{(3)}+2 j_{2 n+1}^{(3)}-4\right)$.

\section{Sum formulas of Generalized Tribonacci Numbers with Negative Subscripts}

The following Theorem presents some linear summing formulas (identities) of generalized Tribonacci numbers with negative subscripts.

Theorem 3.1. For $n \geq 1$, we have the following formulas:

(a) (Sum of the generalized Tribonacci numbers with negative indices) If $r+s+t-1 \neq 0$, then

$$
\sum_{k=1}^{n} W_{-k}=\frac{-(r+s+t) W_{-n-1}-(s+t) W_{-n-2}-t W_{-n-3}+W_{2}+(1-r) W_{1}+(1-r-s) W_{0}}{r+s+t-1} .
$$

(b) If $(r+s+t-1)(r-s+t+1) \neq 0$ then

$$
\sum_{k=1}^{n} W_{-2 k}=\frac{-(r+t) W_{-2 n+1}+\left(r^{2}+r t+s-1\right) W_{-2 n}+(s t-t) W_{-2 n-1}+(1-s) W_{2}+(t+r s) W_{1}+\left(1-r t-2 s-r^{2}+s^{2}\right) W_{0}}{(r+s+t-1)(r-s+t+1)}
$$

and

$$
\sum_{k=1}^{n} W_{-2 k+1}=\frac{(s-1) W_{-2 n+1}-(t+r s) W_{-2 n}-\left(t^{2}+r t\right) W_{-2 n-1}+(r+t) W_{2}+\left(1-r^{2}-r t-s\right) W_{1}+(t-s t) W_{0}}{(r+s+t-1)(r-s+t+1)} .
$$


(c) If $(r+s+t-1)(r-s+t+1) \neq 0 \wedge r+t=0 \wedge s \neq 1$ then

$$
\sum_{k=1}^{n} W_{-2 k}=\frac{-W_{-2 n}-t W_{-2 n-1}+W_{2}+t W_{1}+(1-s) W_{0}}{s-1}
$$

and

$$
\sum_{k=1}^{n} W_{-2 k+1}=\frac{1}{s-1}\left(-W_{-2 n+1}-t W_{-2 n}+W_{1}+t W_{0}\right)
$$

Note that $(c)$ is a special case of $(b)$.

Proof.

(a) Using the recurrence relation

$$
W_{-n+3}=r W_{-n+2}+s W_{-n+1}+t W_{-n} \Rightarrow W_{-n}=-\frac{s}{t} W_{-(n-1)}-\frac{r}{t} W_{-(n-2)}+\frac{1}{t} W_{-(n-3)}
$$

i.e.

$$
t W_{-n}=W_{-n+3}-r W_{-n+2}-s W_{-n+1}
$$

or

$$
W_{-n}=\frac{1}{t} W_{-n+3}-\frac{r}{t} W_{-n+2}-\frac{s}{t} W_{-n+1}
$$

we obtain

$$
\begin{aligned}
t W_{-n} & =W_{-n+3}-r W_{-n+2}-s W_{-n+1} \\
t W_{-n+1} & =W_{-n+4}-r W_{-n+3}-s W_{-n+2} \\
t W_{-n+2} & =W_{-n+5}-r W_{-n+4}-s W_{-n+3} \\
\vdots & \\
t W_{-2} & =W_{1}-r \times W_{0}-s \times W_{-1} \\
t W_{-1} & =W_{2}-r \times W_{1}-s \times W_{0} .
\end{aligned}
$$

If we add the above equations by side by, we get

$$
\sum_{k=1}^{n} W_{-k}=\frac{-\left(r W_{-n-1}+s\left(W_{-n-1}+W_{-n-2}\right)+t\left(W_{-n-1}+W_{-n-2}+W_{-n-3}\right)-W_{2}+(r-1) W_{1}+(r+s-1) W_{0}\right)}{r+s+t-1} .
$$

(b) and (c) Using the recurrence relation

$$
W_{-n+3}=r W_{-n+2}+s W_{-n+1}+t W_{-n}
$$

i.e.

$$
s W_{-n+1}=W_{-n+3}-r W_{-n+2}-t W_{-n}
$$

we obtain

$$
\begin{aligned}
s W_{-2 n+1} & =W_{-2 n+3}-r W_{-2 n+2}-t W_{-2 n} \\
s W_{-2 n+3} & =W_{-2 n+5}-r W_{-2 n+4}-t W_{-2 n+2} \\
\vdots & \\
s W_{-3} & =W_{-1}-r W_{-2}-t W_{-4} \\
s W_{-1} & =W_{1}-r W_{0}-t W_{-2} .
\end{aligned}
$$

If we add the equations by side by, we get

$$
s \sum_{k=1}^{n} W_{-2 k+1}=\left(-W_{-2 n+1}+W_{1}+\sum_{k=1}^{n} W_{-2 k+1}\right)-r\left(-W_{-2 n}+W_{0}+\sum_{k=1}^{n} W_{-2 k}\right)-t\left(\sum_{k=1}^{n} W_{-2 k}\right) .
$$

Similarly, using the recurrence relation

$$
W_{-n+3}=r W_{-n+2}+s W_{-n+1}+t W_{-n}
$$

i.e.

$$
s W_{-n+1}=W_{-n+3}-r W_{-n+2}-t W_{-n}
$$


we obtain

$$
\begin{aligned}
s W_{-2 n} & =W_{-2 n+2}-r W_{-2 n+1}-t W_{-2 n-1} \\
s W_{-2 n+2} & =W_{-2 n+4}-r W_{-2 n+3}-t W_{-2 n+1} \\
\vdots & \\
s W_{-6} & =W_{-4}-r W_{-5}-t W_{-7} \\
s W_{-4} & =W_{-2}-r W_{-3}-t W_{-5} \\
s W_{-2} & =W_{0}-r W_{-1}-t W_{-3} .
\end{aligned}
$$

If we add the above equations by side by, we get

$$
s \sum_{k=1}^{n} W_{-2 k}=\left(-W_{-2 n}+W_{0}+\sum_{k=1}^{n} W_{-2 k}\right)-r\left(\sum_{k=1}^{n} W_{-2 k+1}\right)-t\left(W_{-2 n-1}-W_{-1}+\sum_{k=1}^{n} W_{-2 k+1}\right) .
$$

Since

$$
W_{-1}=\left(-\frac{s}{t} W_{0}-\frac{r}{t} W_{1}+\frac{1}{t} W_{2}\right)
$$

it follows that

$$
s \sum_{k=1}^{n} W_{-2 k}=\left(-W_{-2 n}+W_{0}+\sum_{k=1}^{n} W_{-2 k}\right)-r\left(\sum_{k=1}^{n} W_{-2 k+1}\right)-t\left(W_{-2 n-1}-\left(-\frac{s}{t} W_{0}-\frac{r}{t} W_{1}+\frac{1}{t} W_{2}\right)+\sum_{k=1}^{n} W_{-2 k+1}\right) .
$$

Then, solving system (3.1)-(3.2) the required results of (b) and (c) follow.

Note that (c) of the above theorem can be written as follows: If $r+t=0 \wedge s \neq 1$ then

$$
\sum_{k=1}^{n} W_{-2 k}=\frac{-W_{-2 n}+r W_{-2 n-1}+W_{2}-r W_{1}+(1-s) W_{0}}{s-1}
$$

and

$$
\sum_{k=1}^{n} W_{-2 k}=\frac{-W_{-2 n}+r W_{-2 n-1}+W_{2}-r W_{1}+(1-s) W_{0}}{s-1} .
$$

Next, we present several sum formulas (identities).

Taking $r=s=t=1$ in Theorem 3.1 (a) and (b), we obtain the following Proposition.

Proposition 3.2. If $r=s=t=1$ then for $n \geq 1$ we have the following formulas:

(a) $\sum_{k=1}^{n} W_{-k}=\frac{1}{2}\left(-3 W_{-n-1}-2 W_{-n-2}-W_{-n-3}+W_{2}-W_{0}\right)$.

(b) $\sum_{k=1}^{n} W_{-2 k}=\frac{1}{2}\left(-W_{-2 n+1}+W_{-2 n}+W_{1}-W_{0}\right)$.

(c) $\sum_{k=1}^{n} W_{-2 k+1}=\frac{1}{2}\left(-W_{-2 n}-W_{-2 n-1}+W_{2}-W_{1}\right)$.

From the above Proposition, we have the following Corollary which gives linear sum formulas of Tribonacci numbers (take $W_{n}=T_{n}$ with $\left.T_{0}=0, T_{1}=1, T_{2}=1\right)$.

Corollary 3.3. For $n \geq 1$, Tribonacci numbers have the following properties.

(a) $[13] \sum_{k=1}^{n} T_{-k}=\frac{1}{2}\left(-3 T_{-n-1}-2 T_{-n-2}-T_{-n-3}+1\right)$.

(b) $\sum_{k=1}^{n} T_{-2 k}=\frac{1}{2}\left(-T_{-2 n+1}+T_{-2 n}+1\right)$.

(c) $\sum_{k=1}^{n} T_{-2 k+1}=\frac{1}{2}\left(-T_{-2 n}-T_{-2 n-1}\right)$.

Taking $W_{n}=K_{n}$ with $K_{0}=3, K_{1}=1, K_{2}=3$ in the above Proposition, we have the following Corollary which gives linear sum formulas of Tribonacci-Lucas numbers.

Corollary 3.4. For $n \geq 1$, Tribonacci-Lucas numbers have the following properties:

(a) $\sum_{k=1}^{n} K_{-k}=\frac{1}{2}\left(-3 K_{-n-1}-2 K_{-n-2}-K_{-n-3}\right)$.

(b) $\sum_{k=1}^{n} K_{-2 k}=\frac{1}{2}\left(-K_{-2 n+1}+K_{-2 n}-2\right)$.

(c) $\sum_{k=1}^{n} K_{-2 k+1}=\frac{1}{2}\left(-K_{-2 n}-K_{-2 n-1}+2\right)$.

Taking $r=2, s=1, t=1$ in Theorem 3.1 (a) and (b), we obtain the following Proposition.

Proposition 3.5. If $r=2, s=1, t=1$ then for $n \geq 1$ we have the following formulas:

(a) $\sum_{k=1}^{n} W_{-k}=\frac{1}{3}\left(-4 W_{-n-1}-2 W_{-n-2}-W_{-n-3}+W_{2}-W_{1}-2 W_{0}\right)$.

(b) $\sum_{k=1}^{n} W_{-2 k}=\frac{1}{3}\left(-W_{-2 n+1}+2 W_{-2 n}+W_{1}-2 W_{0}\right)$.

(c) $\sum_{k=1}^{n} W_{-2 k+1}=\frac{1}{3}\left(-W_{-2 n}-W_{-2 n-1}+W_{2}-2 W_{1}\right)$. 
From the last Proposition, we have the following Corollary which gives linear sum formulas of third-order Pell numbers (take $W_{n}=P_{n}^{(3)}$ with $\left.P_{0}^{(3)}=0, P_{1}^{(3)}=1, P_{2}^{(3)}=2\right)$.

Corollary 3.6. For $n \geq 1$, third-order Pell numbers have the following properties.

(a) $\sum_{k=1}^{n} P_{-k}^{(3)}=\frac{1}{3}\left(-4 P_{-n-1}^{(3)}-2 P_{-n-2}^{(3)}-P_{-n-3}^{(3)}+1\right)$.

(b) $\sum_{k=1}^{n} P_{-2 k}^{(3)}=\frac{1}{3}\left(-P_{-2 n+1}^{(3)}+2 P_{-2 n}^{(3)}+1\right)$.

(c) $\sum_{k=1}^{n} P_{-2 k+1}^{(3)}=\frac{1}{3}\left(-P_{-2 n}^{(3)}-P_{-2 n-1}^{(3)}\right)$.

Taking $W_{n}=Q_{n}^{(3)}$ with $Q_{0}^{(3)}=3, Q_{1}^{(3)}=2, Q_{2}^{(3)}=6$ in the last Proposition, we have the following Corollary which gives linear sum formulas of third-order Pell-Lucas numbers.

Corollary 3.7. For $n \geq 1$, third-order Pell-Lucas numbers have the following properties.

(a) $\sum_{k=1}^{n} Q_{-k}^{(3)}=\frac{1}{3}\left(-4 Q_{-n-1}^{(3)}-2 Q_{-n-2}^{(3)}-Q_{-n-3}^{(3)}-2\right)$.

(b) $\sum_{k=1}^{n} Q_{-2 k}^{(3)}=\frac{1}{3}\left(-Q_{-2 n+1}^{(3)}+2 Q_{-2 n}^{(3)}-4\right)$.

(c) $\sum_{k=1}^{n} Q_{-2 k+1}^{(3)}=\frac{1}{3}\left(-Q_{-2 n}^{(3)}-Q_{-2 n-1}^{(3)}+2\right)$.

From the last Proposition, we have the following Corollary which presents linear sum formulas of third-order modified Pell numbers (take $W_{n}=E_{n}^{(3)}$ with $\left.E_{0}^{(3)}=0, E_{1}^{(3)}=1, E_{2}^{(3)}=1\right)$.

Corollary 3.8. For $n \geq 1$, third-order modified Pell numbers have the following properties.

(a) $\sum_{k=1}^{n} E_{-k}^{(3)}=\frac{1}{3}\left(-4 E_{-n-1}^{(3)}-2 E_{-n-2}^{(3)}-E_{-n-3}^{(3)}\right)$.

(b) $\sum_{k=1}^{n} E_{-2 k}^{(3)}=\frac{1}{3}\left(-E_{-2 n+1}^{(3)}+2 E_{-2 n}^{(3)}+1\right)$.

(c) $\sum_{k=1}^{n} E_{-2 k+1}^{(3)}=\frac{1}{3}\left(-E_{-2 n}^{(3)}-E_{-2 n-1}^{(3)}-1\right)$.

Taking $r=0, s=1, t=1$ in Theorem 3.1 (a) and (b), we obtain the following Proposition.

Proposition 3.9. If $r=0, s=1, t=1$ then for $n \geq 1$ we have the following formulas:

(a) $\sum_{k=1}^{n} W_{-k}=-2 W_{-n-1}-2 W_{-n-2}-W_{-n-3}+W_{2}+W_{1}$.

(b) $\sum_{k=1}^{n} W_{-2 k}=-W_{-2 n+1}+W_{1}$.

(c) $\sum_{k=1}^{n} W_{-2 k+1}=-W_{-2 n}-W_{-2 n-1}+W_{2}$.

Taking $W_{n}=P_{n}$ with $P_{0}=1, P_{1}=1, P_{2}=1$ in the last Proposition, we have the following Corollary which gives linear sum formulas of Padovan numbers.

Corollary 3.10. For $n \geq 1$, Padovan numbers have the following properties.

(a) $\sum_{k=1}^{n} P_{-k}=-2 P_{-n-1}-2 P_{-n-2}-P_{-n-3}+2$.

(b) $\sum_{k=1}^{n} P_{-2 k}=-P_{-2 n+1}+1$.

(c) $\sum_{k=1}^{n} P_{-2 k+1}=-P_{-2 n}-P_{-2 n-1}+1$.

From the last Proposition, we have the following Corollary which presents linear sum formulas of Perrin numbers (take $W_{n}=E_{n}$ with $E_{0}=3, E=0, E_{2}=2$ ).

Corollary 3.11. For $n \geq 1$, Perrin numbers have the following properties.

(a) $\sum_{k=1}^{n} E_{-k}=-2 E_{-n-1}-2 E_{-n-2}-E_{-n-3}+2$.

(b) $\sum_{k=1}^{n} E_{-2 k}=-E_{-2 n+1}$.

(c) $\sum_{k=1}^{n} E_{-2 k+1}=-E_{-2 n}-E_{-2 n-1}+2$.

Taking $W_{n}=S_{n}$ with $S_{0}=0, S_{1}=0, S_{2}=1$ in the last Proposition, we have the following Corollary which gives linear sum formulas of Padovan-Perrin numbers.

Corollary 3.12. For $n \geq 1$, Padovan-Perrin numbers have the following properties.

(a) $\sum_{k=1}^{n} S_{-k}=-2 S_{-n-1}-2 S_{-n-2}-S_{-n-3}+1$.

(b) $\sum_{k=1}^{n} S_{-2 k}=-S_{-2 n+1}$.

(c) $\sum_{k=1}^{n} S_{-2 k+1}=-S_{-2 n}-S_{-2 n-1}+1$.

If $r=0, s=2, t=1$ then $(r+s+t-1)(r-s+t+1)=0$ so we can't use Theorem 3.1 (b) and (c). In other words, the method of the proof Theorem 3.1 (b) and (c) can't be used to find $\sum_{k=0}^{n} W_{2 k}$ and $\sum_{k=0}^{n} W_{2 k+1}$. Therefore we need another method to find them which is given in the following Theorem.

Theorem 3.13. If $r=0, s=2, t=1$ then for $n \geq 1$ we have the following formulas:

(a) $\sum_{k=1}^{n} W_{-k}=\frac{1}{2}\left(-3 W_{-n-1}-3 W_{-n-2}-W_{-n-3}+W_{2}+W_{1}-W_{0}\right)$.

(b) $\sum_{k=1}^{n} W_{-2 k}=-W_{-2 n+1}+W_{-2 n}+\left(W_{1}-W_{0}\right)+\left(W_{2}-W_{1}-W_{0}\right) n$.

(c) $\sum_{k=1}^{n} W_{-2 k+1}=\frac{1}{2}\left(W_{-2 n+1}-3 W_{-2 n}-W_{-2 n-1}+\left(W_{2}-W_{1}+W_{0}\right)+2\left(-W_{2}+W_{1}+W_{0}\right) n\right)$. 
Proof.

(a) Taking $r=0, s=2, t=1$ in Theorem 3.1 (a) we obtain (a).

(b) and (c) Proof can be done as in the proof of Theorem 2.13. Induction also can be used for the proof.

From the last Theorem, we have the following Corollary which gives sum formula of Pell-Padovan numbers (take $W_{n}=R_{n}$ with $R_{0}=1, R=$ $\left.1, R_{2}=1\right)$.

Corollary 3.14. For $n \geq 1$, Pell-Padovan numbers have the following property:

(a) $\sum_{k=1}^{n} R_{-k}=\frac{1}{2}\left(-3 R_{-n-1}-3 R_{-n-2}-R_{-n-3}+1\right)$.

(b) $\sum_{k=1}^{n} R_{-2 k}=-R_{-2 n+1}+R_{-2 n}-n$.

(c) $\sum_{k=1}^{n} R_{-2 k+1}=\frac{1}{2}\left(R_{-2 n+1}-3 R_{-2 n}-R_{-2 n-1}+1+2 n\right)$.

Taking $W_{n}=C_{n}$ with $C_{0}=3, C=0, C_{2}=2$ in the last Theorem, we have the following Corollary which gives sum formulas of Pell-Perrin numbers.

Corollary 3.15. For $n \geq 1$, Pell-Perrin numbers have the following property:

(a) $\sum_{k=1}^{n} C_{-k}=\frac{1}{2}\left(-3 C_{-n-1}-3 C_{-n-2}-C_{-n-3}-1\right)$

(b) $\sum_{k=1}^{n} C_{-2 k}=-C_{-2 n+1}+C_{-2 n}-3-n$

(c) $\sum_{k=1}^{n} C_{-2 k+1}=\frac{1}{2}\left(C_{-2 n+1}-3 C_{-2 n}-C_{-2 n-1}+5+2 n\right)$

Taking $r=0, s=1, t=2$ in Theorem 3.1 (a) and (b), we obtain the following Proposition.

Proposition 3.16. If $r=0, s=1, t=2$ then for $n \geq 1$ we have the following formulas:

(a) $\sum_{k=1}^{n} W_{-k}=\frac{1}{2}\left(-3 W_{-n-1}-3 W_{-n-2}-2 W_{-n-3}+W_{2}+W_{1}\right)$.

(b) $\sum_{k=1}^{n} W_{-2 k}=\frac{1}{2}\left(-W_{-2 n+1}+W_{1}\right)$.

(c) $\sum_{k=1}^{n} W_{-2 k+1}=\frac{1}{2}\left(-W_{-2 n}-2 W_{-2 n-1}+W_{2}\right)$.

From the last Proposition, we have the following Corollary which gives linear sum formulas of Jacobsthal-Padovan numbers (take $W_{n}=Q_{n}$ with $\left.Q_{0}=1, Q_{1}=1, Q_{2}=1\right)$.

Corollary 3.17. For $n \geq 1$, Jacobsthal-Padovan numbers have the following properties.

(a) $\sum_{k=1}^{n} Q_{-k}=\frac{1}{2}\left(-3 Q_{-n-1}-3 Q_{-n-2}-2 Q_{-n-3}+2\right)$.

(b) $\sum_{k=1}^{n} Q_{-2 k}=\frac{1}{2}\left(-Q_{-2 n+1}+1\right)$.

(c) $\sum_{k=1}^{n} Q_{-2 k+1}=\frac{1}{2}\left(-Q_{-2 n}-2 Q_{-2 n-1}+1\right)$.

Taking $W_{n}=D_{n}$ with $D_{0}=3, D_{1}=0, D_{2}=2$ in the last Proposition, we have the following Corollary which gives linear sum formulas of Jacobsthal-Perrin numbers.

Corollary 3.18. For $n \geq 1$, Jacobsthal-Perrin numbers have the following properties.

(a) $\sum_{k=1}^{n} D_{-k}=\frac{1}{2}\left(-3 D_{-n-1}-3 D_{-n-2}-2 D_{-n-3}+2\right)$.

(b) $\sum_{k=1}^{n} D_{-2 k}=\frac{-1}{2} D_{-2 n+1}$.

(c) $\sum_{k=1}^{n} D_{-2 k+1}=\frac{1}{2}\left(-D_{-2 n}-2 D_{-2 n-1}+2\right)$.

Taking $r=1, s=0, t=1$ in Theorem 3.1, we obtain the following Proposition.

Proposition 3.19. If $r=1, s=0, t=1$ then for $n \geq 1$ we have the following formulas:

(a) $\sum_{k=1}^{n} W_{-k}=-2 W_{-n-1}-W_{-n-2}-W_{-n-3}+W_{2}$.

(b) $\sum_{k=1}^{n} W_{-2 k}=\frac{1}{3}\left(-2 W_{-2 n+1}+W_{-2 n}-W_{-2 n-1}+W_{2}+W_{1}-W_{0}\right)$.

(c) $\sum_{k=1}^{n} W_{-2 k+1}=\frac{1}{3}\left(-W_{-2 n+1}-W_{-2 n}-2 W_{-2 n-1}+2 W_{2}-W_{1}+W_{0}\right)$.

From the above Proposition, we have the following Corollary which gives linear sum formulas of Narayana numbers (take $W_{n}=N_{n}$ with $\left.N_{0}=0, N_{1}=1, N_{2}=1\right)$.

Corollary 3.20. For $n \geq 1$, Narayana numbers have the following properties.

(a) $\sum_{k=1}^{n} N_{-k}=-2 N_{-n-1}-N_{-n-2}-N_{-n-3}+1$.

(b) $\sum_{k=1}^{n} N_{-2 k}=\frac{1}{3}\left(-2 N_{-2 n+1}+N_{-2 n}-N_{-2 n-1}+2\right)$.

(c) $\sum_{k=1}^{n} N_{-2 k+1}=\frac{1}{3}\left(-N_{-2 n+1}-N_{-2 n}-2 N_{-2 n-1}+1\right)$.

Taking $r=1, s=1, t=2$ in Theorem 3.1, we obtain the following Proposition.

Proposition 3.21. If $r=1, s=1, t=2$ then for $n \geq 1$ we have the following formulas:

(a) $\sum_{k=1}^{n} W_{-k}=\frac{1}{3}\left(-4 W_{-n-1}-3 W_{-n-2}-2 W_{-n-3}+W_{2}-W_{0}\right)$.

(b) $\sum_{k=1}^{n} W_{-2 k}=\frac{1}{3}\left(-W_{-2 n+1}+W_{-2 n}+W_{1}-W_{0}\right)$.

(c) $\sum_{k=1}^{n} W_{-2 k+1}=\frac{1}{3}\left(-W_{-2 n}-2 W_{-2 n-1}+W_{2}-W_{1}\right)$. 
Taking $W_{n}=J_{n}$ with $J_{0}=0, J_{1}=1, J_{2}=1$ in the last Proposition, we have the following Corollary which gives linear sum formulas of third order Jacobsthal numbers.

Corollary 3.22. For $n \geq 1$, third order Jacobsthal numbers have the following properties.

(a) $\sum_{k=1}^{n} J_{-k}^{(3)}=\frac{1}{3}\left(-4 J_{-n-1}^{(3)}-3 J_{-n-2}^{(3)}-2 J_{-n-3}^{(3)}+1\right)$.

(b) $\sum_{k=1}^{n} J_{-2 k}^{(3)}=\frac{1}{3}\left(-J_{-2 n+1}^{(3)}+J_{-2 n}^{(3)}+1\right)$.

(c) $\sum_{k=1}^{n} J_{-2 k+1}^{(3)}=\frac{1}{3}\left(-J_{-2 n}^{(3)}-2 J_{-2 n-1}^{(3)}\right)$.

From the last Proposition, we have the following Corollary which gives linear sum formulas of third order Jacobsthal-Lucas numbers (take $W_{n}=j_{n}^{(3)}$ with $j_{0}^{(3)}=2, j_{1}^{(3)}=1, j_{2}^{(3)}=5$ ).

Corollary 3.23. For $n \geq 1$, third order Jacobsthal-Lucas numbers have the following properties.

(a) $\sum_{k=1}^{n} j_{-k}^{(3)}=\frac{1}{3}\left(-4 j_{-n-1}^{(3)}-3 j_{-n-2}^{(3)}-2 j_{-n-3}^{(3)}+3\right)$.

(b) $\sum_{k=1}^{n} j_{-2 k}^{(3)}=\frac{1}{3}\left(-j_{-2 n+1}^{(3)}+j_{-2 n}^{(3)}-1\right)$.

(c) $\sum_{k=1}^{n} j_{-2 k+1}^{(3)}=\frac{1}{3}\left(-j_{-2 n}^{(3)}-2 j_{-2 n-1}^{(3)}+4\right)$.

\section{References}

[1] K. Adegoke, Summation identities involving Padovan and Perrin numbers, (2019), arXiv:1812.03241v2 [math.CO].

[2] I. Bruce, A modified Tribonacci sequence, Fibonacci Quart., 22(3) (1984), 244-246.

[3] M. Catalani, Identities for Tribonacci-related sequences, (2002), arXiv:0209179 [math.CO]

[4] G. Cerda-Morales, On a generalization for Tribonacci quaternions, Mediterr. J. Math., 14(239) (2017), 1-12

[5] E. Choi, Modular Tribonacci numbers by matrix method, J. Korean Soc. Math. Educ. Ser. B: Pure Appl. Math. 20(3) (2013), 207-221.

[6] C.K. Cook, Bacon, M.R.: Some identities for Jacobsthal and Jacobsthal-Lucas numbers satisfying higher order recurrence relations. Ann. Math. Inform., 41 (2013), 27-39.

[7] M. Elia, Derived Sequences, The Tribonacci recurrence and cubic forms, Fibonacci Quart., 39(2) (2001), 107-115.

[8] R. Frontczak, Sums of Tribonacci and Tribonacci-Lucas numbers, Int. J. Math. Anal. 12(1) (2018), 19-24.

[9] H. Gökbaş, H. Köse, Some sum formulas for products of Pell and Pell-Lucas numbers, Int. J. Adv. Appl. Math. and Mech. 4(4) (2017), 1-4.

[10] R.T. Hansen, General identities for linear Fibonacci and Lucas summations, Fibonacci Quart., 16(2) (1978), 121-28.

[11] T. Koshy, Fibonacci and Lucas Numbers with Applications, A Wiley-Interscience Publication, New York, 2001.

[12] T. Koshy, Pell and Pell-Lucas Numbers with Applications, Springer, New York, 2014.

[13] K. Kuhapatanakul, L. Sukruan, The Generalized Tribonacci numbers with negative subscripts, Integer, 14 (2014), 1-6.

[14] P. Y. Lin, De Moivre-type identities for the Tribonacci numbers, Fibonacci Quart., 26 (1988), 131-134.

[15] S. Pethe, Some Identities for Tribonacci sequences, Fibonacci Quart., 26(2) (1988), 144-151.

[16] T. Parpar, k’ncı mertebeden rekürans bă̆ıntısının özellikleri ve bazı uygulamaları, Yüksek Lisans Tezi, Selçuk Üniversitesi, Fen Bilimleri Enstitüsü,

[17] A. Scott, T. Delaney, Jr. V. Hoggatt, The Tribonacci sequence, Fibonacci Quart., 15(3) (1977), 193-200.

[18] A.G Shannon, A.F. Horadam, Some properties of third-order recurrence relations, Fibonacci Quart., 10(2) (1972), $135-146$.

[19] A. Shannon, Tribonacci numbers and Pascal's pyramid, Fibonacci Quart., 15(3) (1977), pp. 268 and 275.

[20] N.J.A. Sloane, The on-line encyclopedia of integer sequences. Available: http://oeis.org/

[21] Y. Soykan, Matrix sequences of Tribonacci and Tribonacci-Lucas numbers, (2018), arXiv:1809.07809v1 [math.NT] .

[22] Y. Soykan, Linear summing formulas of generalized Pentanacci and Gaussian generalized Pentanacci numbers, Journal of Advanced in Mathematics and Computer Science, 33(3) (2019), 1-14.

[23] Y. Soykan, On summing formulas of generalized Hexanacci and Gaussian generalized Hexanacci numbers, Asian Research Journal of Mathematics, 14(4) (2019), 1-14.

[24] Y. Soykan, On summing formulas for generalized Fibonacci and Gaussian generalized Fibonacci numbers, Advances in Research, 20(2) (2019), 1-15.

[25] Y. Soykan, On generalized Third-Order Pell numbers, Asian Journal of Advanced Research and Reports, 6(1) (2019), 1-18.

[26] W. Spickerman, Binet's formula for the Tribonacci sequence, Fibonacci Quart., 20 (1982), 118-120.

[27] C. C. Yalavigi, A Note on 'Another Generalized Fibonacci Sequence', Math. Student. 39 (1971), $407-408$.

[28] C. C. Yalavigi, Properties of Tribonacci numbers, Fibonacci Quart., 10(3) (1972), 231-246.

[29] N. Yilmaz, N. Taskara, Tribonacci and Tribonacci-Lucas numbers via the determinants of special matrices, Appl. Math. Sci., 8(39) (2014), 1947-1955.

[30] M. E. Waddill, Using matrix techniques to establish properties of a generalized Tribonacci sequence (in Applications of Fibonacci Numbers, Volume 4 G. E. Bergum et al., eds.). Kluwer Academic Publishers. Dordrecht, The Netherlands: pp. 299-308, 1991.

[31] M. E. Waddill, The Tetranacci sequence and generalizations, Fibonacci Quart., (1992), 9-20. 